\title{
T-cell resistance to regulation in RRMS linked to IL-6 pathway
}

Multiple sclerosis (MS) is driven by neuroinflammation, but the mechanisms underlying failure to control the autoimmune response have been unclear. Now, researchers from the Benaroya Research Institute in Seattle, WA, USA, report that $\mathrm{CD}^{+}$effector $\mathrm{T}\left(\mathrm{T}_{\mathrm{EFF}}\right)$ cells from patients with relapsing-remitting MS (RRMS) are resistant to suppression by T-regulatory $\left(\mathrm{T}_{\mathrm{REG}}\right)$ cells, and that this mechanism is linked to the IL-6 pathway.

Previous studies suggested that a reduction in the number and function of $\mathrm{T}_{\mathrm{REG}}$ cells caused the failure of immune regulation in RRMS. In other autoimmune diseases such as psoriasis and diabetes, however, resistance of $\mathrm{T}_{\mathrm{EFF}}$ cells to $\mathrm{T}_{\mathrm{REG}}$-mediated suppression has been observed.

"The ability of IL- 6 to cause $\mathrm{T}_{\mathrm{EFF}}$ resistance has already been shown in other autoimmune diseases, and IL-6 is an important contributor to pathology in a mouse model of MS," says Jane Hoyt Buckner, who led the study. Signalling via the IL-6 receptor leads to phosphorylation of signal transducer and activator of transcription 3 (pSTAT3), and variants of the STAT3 gene have been linked to MS risk, but this signalling pathway has not been extensively investigated in RRMS.
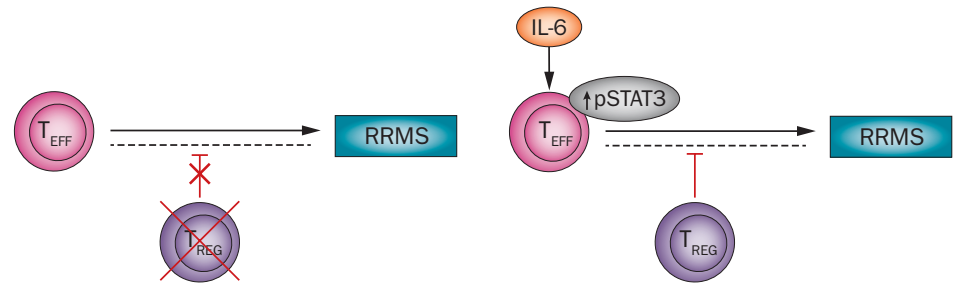

Failure of regulation in RRMS could result from inadequate numbers or function of $T_{\text {REG }}$ cells (left) or from $T_{E F F}$ resistance to suppression, mediated by IL-6 signalling and phosphorylation of STAT3 (right).

"We studied a group of RRMS patients who were not receiving immunological therapies at the time of blood draw, and performed in vitro suppression assays with $\mathrm{T}_{\mathrm{EFF}}$ cells from these individuals while using $\mathrm{T}_{\mathrm{REG}}$ cells from healthy controls," explains Buckner. "This approach enabled us to evaluate the $\mathrm{T}_{\mathrm{EFF}}$ population specifically."

In two independent cohorts (total $n=24$ ), suppression of $\mathrm{T}_{\mathrm{EFF}}$ cells from patients with RRMS was significantly lower than that of $\mathrm{T}_{\mathrm{EFF}}$ cells from healthy controls. After stimulation with IL-6, the percentage of pSTAT3-positive $\mathrm{CD} 4^{+}$cells was higher in the RRMS cohort than in controls, suggesting that $\mathrm{T}_{\mathrm{EFF}}$ cells from patients with RRMS are more responsive to IL-6.

In both groups, a negative correlation was observed between pSTAT3 expression and the level of $\mathrm{T}_{\mathrm{EFF}}$ suppression, and resistance of $\mathrm{T}_{\mathrm{EFF}}$ cells from three individuals with RRMS was reduced by a STAT3 inhibitor.

Collectively, these findings support a role for the IL-6 pathway in regulation of resistance in RRMS. "Furthermore, we found that alterations in $\mathrm{T}_{\mathrm{EFF}}$ resistance were associated with more-aggressive and active disease," says Buckner.

The group now plans to assess IL-6 responses and $\mathrm{T}_{\mathrm{EFF}}$ resistance over the course of disease in a larger RRMS cohort, and to establish whether these parameters change with therapy.

\section{Katy Malpass}

Original article Schneider, A. et al. In active relapsingremitting multiple sclerosis, effector $T$ cell resistance to adaptive $\mathrm{T}_{\text {regs }}$ involves IL-6 mediated signaling. Sci. Transl. Med. 5, 170ra15 (2013) 